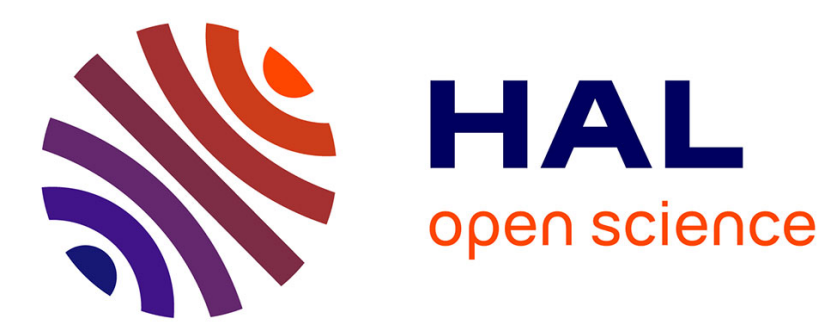

\title{
La modélisation hydraulique comme condition de la résilience des projets d'aménagement urbain en zone inondable?
}

\author{
Mathieu Bonnefond
}

\section{- To cite this version:}

Mathieu Bonnefond. La modélisation hydraulique comme condition de la résilience des projets d'aménagement urbain en zone inondable?. La Houille Blanche - Revue internationale de l'eau, 2018, 3, pp.25-33. 10.1051/lhb/2018029 . hal-03027378

\section{HAL Id: hal-03027378 \\ https://hal.science/hal-03027378}

Submitted on 27 Nov 2020

HAL is a multi-disciplinary open access archive for the deposit and dissemination of scientific research documents, whether they are published or not. The documents may come from teaching and research institutions in France or abroad, or from public or private research centers.
L'archive ouverte pluridisciplinaire $\mathbf{H A L}$, est destinée au dépôt et à la diffusion de documents scientifiques de niveau recherche, publiés ou non, émanant des établissements d'enseignement et de recherche français ou étrangers, des laboratoires publics ou privés. 


\title{
LA MODELISATION HYDRAULIQUE COMME CONDITION DE LA RESILIENCE DES PROJETS D'AMENAGEMENT URBAIN EN ZONE INONDABLE ?
}

\author{
Mathieu BONNEFOND(1) \\ (1) LTSER Zone Atelier Loire - CNRS - Conservatoire National des Arts et Métiers - EA 4630 Laboratoire \\ Géomatique et Foncier - 1 bd Pythagore 72000 Le Mans - mathieu.bonnefond@lecnam.net
}

L'analyse de la modélisation hydraulique à l'épreuve des faits, c'est à dire en ce qu'elle produit en matière de règles et d'action collective locale, de conception et de modalités d'aménagement, permet de mieux appréhender son rôle, de plus en plus important, dans la construction d'une résilience urbaine face au risque inondation. La mobilisation de ces outils de modélisation par les différents acteurs pose les bases d'une résilience technique mais aussi plus globale dans les secteurs aménagés en zone inondable. Les travaux de recherche présentés dans ce papier ont été réalisés dans le cadre du projet PRÉCIEU (Programme RDT) où nous avons cherché à analyser la résilience urbaine à l'épreuve des faits à l'échelle du projet urbain de quartier. Au travers de l'étude de deux cas dans le bassin de la Loire (Angers et Romorantin-Lanthenay), nous montrons en quoi la modélisation hydraulique évolue dans son rôle, de socle de la définition des règles d'aménagement à la condition de la construction de quartier résilient. Nous montrons en quoi cet outil n'est pas restreint à sa dimension technique mais participe de l'action collective locale et en quoi il permet le renouvellement de la prise en charge du risque inondation, d'une approche par la règle vers une approche dynamique par le projet qui permet de dé-compartimenter les acteurs de la ville et de proposer des modalités d'adaptation du territoire au risque inondation.

MOTS CLEFS : Modélisation hydraulique, Résilience urbaine, Risque inondation, Projet d'aménagement urbain

\section{Hydraulic modeling as a condition of resilient urban projects in flood-prone area?}

This paper analyses hydraulic modeling as a proof of the facts, its outputs in urban design process and its importance in local resilience construction More precisely, we focus on the outputs of hydraulic modeling in terms of rules' production, collective action, urban design, etc. At the level of urban projects, this approach by the facts enables to identify the role of modeling in the implementation of urban resilience in face of the flood risk. Indeed, the mobilization of these technical tools by stakeholders sets the foundations of technical resilience or even global resilience in a flood-prone area. This research was carried out within the framework of the «PRÉCIEU » project (RTD french program). In this project, we sought to analyze urban resilience as a proof of the facts at the scale of the urban estate's project. In this paper, we show, through the study of two cases in the Loire basin (Angers et Romorantin), how hydraulic modeling evolves in its role, from the foundation of the definition of the rules of development to a necessary condition for the construction of resilient neighborhoods. Then we explain how this tool is not restricted to its technical dimension but also participates to the construction of local collective action. We highlight its role in the negotiation process between government agency, local governement and urbanists. We describe how models contribute to the renewal of flood risk management, from a approach by the rules towards a more dynamic approach focusing on projects, lifting barriers between the various actors of the city and proposing ways to better adapt the urban estates to the flood risk. 


\section{KEY WORDS : Hydraulic modeling, Urban resilience, Flood risk, Urban development} project

\section{INTRODUCTION}

Ce papier s'intéresse à I'utilisation de la modélisation hydraulique ${ }^{1}$ à différents niveaux dans les projets d'aménagement urbain en zone inondable et à son rôle dans la construction d'une résilience du point de vue de la capacité de résistance, d'absorption, de récupération des systèmes urbains face au risque inondation [Serre, 2011] et de l'organisation territoriale [Barroca et al., 2012]. Le papier fait le choix d'une définition volontairement restreinte du terme de résilience, tant celui-ci est parfois « sur-employé » [Reghezza et al., 2012]. Nous interrogerons en quoi la mobilisation de ces outils par les différents acteurs pose les bases d'une résilience technique mais aussi sociale dans les secteurs aménagés en zone inondable, qui constituent aujourd'hui des enjeux fort en matière d'urbanisme [Rode, 2017]. Il s'agit d'analyser la modélisation hydraulique à l'épreuve des faits, c'est à dire en ce qu'elle produit en matière de règles et d'action collective locale, de conception et de modalités d'aménagement. Cette approche a été particulièrement développée dans le cadre du projet PRÉCIEU² où nous avons cherché à analyser la résilience urbaine à l'épreuve des faits, c'est-à-dire comment s'élabore une urbanisation au plus près du risque d'inondation, pensée à l'échelle du projet urbain de quartier. Nous avons notamment pu analyser le rôle de la modélisation hydraulique dans l'élaboration et la négociation de la contrainte d'inondation en lien avec les enjeux urbains, paysagers et architecturaux. Si la technicité des modèles est centrale, la modélisation est interrogée ici au prisme des effets qu'elle produit dans le processus de production de la ville et en quoi elle peut être une des conditions préalables et nécessaires à la résilience urbaine. A I'heure où I'urbanisation des zones inondables pose question [Barroca \& Hubert, 2008], nous faisons ici le choix d'étudier des projets de renouvellement urbain en secteur inondable. Au travers de l'étude de deux cas dans le bassin de la Loire, le quartier Saint-Serge à Angers et le Quartier Matra à Romorantin-Lanthenay, nous montrons en quoi la modélisation hydraulique évolue dans son rôle, d'un socle de la définition des règles d'aménagement à un outil de construction de la résilience urbaine.

\section{DES PROJETS DE RENOUVELLEMENT URBAIN FACE AU RISQUE INONDATION : MODELISER POUR REGULER}

Les sites étudiés correspondent à des projets de renouvellement urbain (Figure 1) en zone inondable pour lequel la modélisation hydraulique a un rôle prépondérant à plusieurs niveaux.

\footnotetext{
1 Outil de calcul numérique qui permet de caractériser l'écoulement de l'eau (hauteurs et vitesses de l'eau) en fonction d'un débit.

2 Le PRojet d'Etudes sur la Contrainte d'Inondation dans les projets urbains en Espaces Urbanisés a été financé dans le cadre du programme RDT, ce projet est coordonné par Mathilde Gralepois et Sylvain Rode.
} 


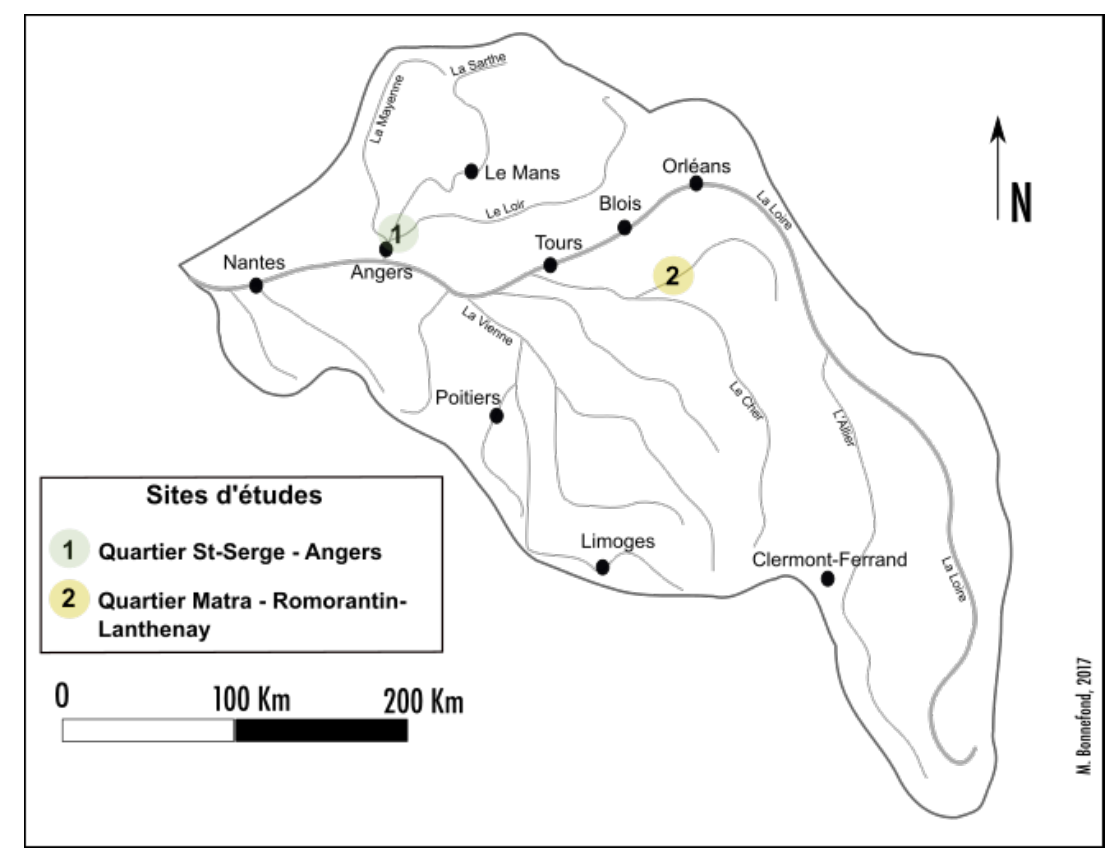

Figure 1 : Localisation des deux sites d'étude

Le site d'Angers St Serge-Thiers Boisnet, était une zone humide de vastes prairies inondables, bordant la Maine dans la partie nord de la ville d'Anger, remblayées au XIXème siècle pour laisser place à un faubourg populaire à la fois marchand et industriel, puis au XXème siècle à une zone d'activité. A partir de 1985, la ville d'Angers réfléchit à la requalification de la zone industrielle et commerciale, stratégique pour la ville et I'agglomération, puisque située favorablement en bord de Maine et à proximité du centre-ville. La présence d'entrepôts et de nombreuses emprises ferroviaires et artisanales offre des potentiels de renouvellement important dans le tissu constitué. Il est alors choisi de réaménager la partie sud du quartier. Deux Zones d'Aménagements Concertés (ZAC) sont mises en place à partir de 1991 avec la volonté de respecter le tissu urbain existant avec un maillage orthogonal. L'emprise du projet était comprise dans une zone inondable identifiée par le Plan des Surfaces Submersibles de la Maine imposant une cote plancher. Lors de la première phase d'aménagement de la ZAC dite «Saint-Serge 2000 » et du démarrage de la ZAC dite «Thiers Boisnet », le quartier a connu un épisode de crue centennale du 22 janvier au 4 février 1995 marquant l'ensemble du bassin de la Maine par son intensité et par l'étendue des dégâts qu'il a provoqué. Des hauteurs d'eau de 50 à $60 \mathrm{~cm}$ ont été constatées par endroit dans ce quartier. Lors de la crue de 1995, quelques constructions avaient déjà débuté. Cette crue a profondément marqué les esprits et a conduit à la rénovation des documents de gestion du risque inondation ainsi qu'une requalification de l'aménagement dans ces ZAC. A partir des années 2000, tirant les enseignements des aménagements réalisés dans la partie sud du quartier, une réflexion plus globale est engagée au travers du projet Angers Rives Nouvelles ${ }^{3}$. Ce projet vise notamment à reconnecter la ville d'Angers avec la Maine notamment par la suppression de la voie expresse sur berges ainsi qu'à accompagner la requalification de la zone d'activité de la partie nord du quartier. Le nouveau projet intègre dès sa conception la problématique de l'inondation.

Le second site se trouve dans l'emprise de l'ancienne usine Matra fermée en 2003. Dès la fin du XIXème siècle, le site alors dénommé Faubourg Saint-Roch est occupé par les manufactures de draperie Normant puis par I'usine Matra Automobile à partir des années 1970. En 2006, la municipalité de RomorantinLanthenay rachète le site dans le but de réaliser un projet de restructuration de grande envergure devant donner naissance à un nouveau quartier. Cette opération avait une double vocation à la fois économique et résidentielle sur les 6 hectares longeant la Sauldre. Le programme prévoyait la construction de nombreux logements et d'équipement ainsi que d'un musée. Le projet dénommé alors Romo 1 porté dans un premier temps par un promoteur se trouve rapidement confronté à des problématiques multiples : conservation de partie d'usine au titre des monuments historiques, pollution des sols et risque inondation. C'est ce dernier qui fait abandonner le projet par le promoteur face aux incertitudes et contraintes à venir liées à l'élaboration d'un Plan de Prévention du Risque inondation (PPRi). La municipalité et l'architecte-urbaniste du projet décident de poursuivre malgré tout en décidant d'intégrer l'inondation au cœur du projet.

Sur ces deux sites, les projets de renouvellement sont confrontés d'abord à la problématique du risque inondation au travers des règles d'urbanisation qui en découlent. Ces règles sont élaborées à partir d'une modélisation des inondations, au sens de la production d'une représentation schématique du processus

3 Le projet Angers Rives Nouvelles a été largement requalifié au moment de I'alternance municipale en 2014, avec l'abandon du principe de la suppression de la voie sur berges. Le nouveau projet se dénomme Angers Cœur de Maine. Nous avons fait le choix d'étudier spécifiquement ce projet avant son abandon car il incarne une nouvelle approche de la prise en compte de l'inondation par le projet urbain. 
hydraulique (hauteur et vitesse de l'eau en fonction d'un débit) et de sa traduction spatiale (cartographie des aléas) qui fonde le règlement du PPRi. Si la hauteur est bien appréhendée dans cette démarche, la question de la vitesse des écoulements l'est moins, notamment au niveau des seuils critiques de vitesse en fonction du bâti. L'objectif étant d'une manière générale d'élaborer un règlement visant à limiter les effets de l'inondation sur les bâtiments (cote de plancher), les effets de ces derniers sur le phénomène d'inondation (les règles d'emprise), et enfin la protection des équipements. Ce processus conduit nécessairement à une réduction de la complexité des phénomènes. Pour cela, les services de l'État définissent la cote des Plus Hautes Eaux Connues (PHEC) et les vitesses d'écoulement qui servent de base à l'élaboration de ces règles de contrôle d'usage. La circulaire du 24 janvier 1994, qui pose les règles d'élaboration des PPRi, précise que l'événement de référence à retenir est, conventionnellement, « la plus forte crue connue et, dans le cas où celle-ci serait plus faible qu'une crue centennale, cette dernière ».

La modélisation hydraulique, même rudimentaire, est I'outil qui va fonder la définition des aléas. Les services de l'Etat déterminent la crue historique de référence à partir d'une étude historique. Cette crue de référence se base sur un phénomène historique connu et va permettre de définir l'aléa, c'est à dire les hauteurs de submersion et les vitesses d'écoulement. Malgré les observations historiques (laisses de crue, station hydrologique, etc.), du fait des aménagements dans les zones inondées intervenus depuis la survenue de l'épisode de référence, il peut être nécessaire de réaliser d'une modélisation hydraulique plus ou moins complexe à partir des données topographiques actuelles. La précision et la résolution des modèles numériques de terrain (la topographie) sont déterminantes dans l'élaboration des cartes de hauteurs de submersion. Les services de l'Etat peuvent commanditer un bureau d'étude pour réaliser une modélisation hydraulique poussée (Modèle 2D) mais coûteuse. Si le principe qui fonde cette approche en termes de surface submersible date des années 30, leur réalisation a suivi les innovations technologiques et notamment le développement de la modélisation numérique. Ces évolutions permettent aujourd'hui de mieux définir les écoulements et notamment les vitesses (trajets préférentiels, effets dynamiques des éléments bâtis et de la morphologie urbaine, etc.) ainsi que des effets hydrodynamiques telles que les ruptures de digue (effet de vague). Les PPRi sur les deux sites étudiés ont été réalisés récemment, soit concomitamment à l'élaboration du projet comme à Romorantin-Lanthenay, soit en parallèle de son émergence comme à Angers. Ainsi, sur le site Matra, si le PPRi de la Sauldre est définitivement approuvé le 02 octobre 2015, son intégration dans le projet et ses premiers aménagements s'est fait largement en amont. Cela démontre une démarche particulière des porteurs de projet. La démarche a consisté à concevoir un quartier en mettant au centre l'inondation et en se basant sur la modélisation hydraulique dans ce cadre (Cf. infra). Dans la mesure où l'émergence du PPRi et celle du projet sont à peu près concomitantes, un règlement spécifique sur ces quartiers en aléa faible est alors créé (zone $\mathrm{B}$ ). Ce classement en zone Bleu permet la construction et les aménagements sous conditions de ces quartiers.

In fine, les PHEC et les vitesses d'écoulement correspondent plus à des niveaux conventionnels qui prennent une valeur réglementaire avec l'approbation du PPRi. Si la modélisation des hauteurs et des vitesses d'écoulement permet d'améliorer la cartographie de l'aléa, une certaine incertitude demeure. La pertinence de cette cote au même titre que la définition de l'occurrence des phénomènes de crue (période de retour) reste critiquable, alors même qu'elle fonde la définition des règles [Bernardara et Barroca, 2005]. L'actualité récente, avec les crues de mai-juin 2016, I'a bien démontré ${ }^{4}$.Toutefois, le règlement du PPRi peut intégrer des dispositions particulières prenant en compte une certaine marge d'erreur dans la définition des PHEC et des vitesses d'écoulement de l'eau, comme le principe d'une cote plancher $50 \mathrm{~cm}$ au-dessus des PHEC. Cette marge permet d'intégrer les erreurs et incertitudes liées au processus de définition des PHEC. Ce principe, comme nous le verrons plus loin, peut se révéler salutaire.

La traduction de l'aléa en règles pose aussi des questions d'échelle. En effet, les règles du PPRi sont produites le plus souvent pour une application à l'échelle du bâtiment, le contraignant notamment dans sa cote du plancher habitable ainsi que dans son emprise au sol. L'échelle de la règle s'inscrit dans une approche administrative, celle de la procédure de permis de construire, cela pose question quand l'échelle de conception est celle du quartier, comme nous le rappelle Éric Daniel Lacombe lors d'une visite du quartier Matra : «il y a aussi une notion d'échelle dans la cohérence de la règle. Ce qui est applicable pour un projet de 6 ha ne l'est pas pour un projet de $600 \mathrm{~m}^{2}$, alors que les contraintes sont les mêmes. » (Daniel Lacombe, 2016). Ce qui amène les opérateurs de ces projets à négocier l'application des règles. Ces dynamiques dans une certaine mesure sont révélatrices de la primauté du projet sur la règle.

\footnotetext{
${ }^{4}$ Cf. article de Laurence Madoui « inondations : quand les modèles hydrologiques prennent l'eau » publié dans le dossier « Inondations : apprendre à prévenir et à gérer le risque » de la Gazette des communes du $19 / 07 / 2016$
} 


\section{QUAND LA MODÉLISATION HYDRAULIQUE S'INVITE AU COEUR DES PROJETS D'AMENAGEMENT URBAIN : ENTRE CONCEPTION ET NÉGOCIATION}

La modélisation hydraulique a été mobilisée dans le cadre de la conception des projets de la Cité Fluviale Matra à Romorantin-Lanthenay et d'Angers Rives Nouvelles. Elle est alors l'expression de la primauté du projet sur le contrôle de l'usage du sol. Des concepteurs et opérateurs d'aménagement en zone inondable, collectivités locales, architecte-urbanistes, ont été ainsi amenés à faire réaliser, par des cabinets spécialisés, des modèles hydrauliques permettant d'évaluer l'effet de leur aménagement. Cette modélisation souvent plus poussée et donc plus onéreuse que celle qui fonde la planification des usages, en plus de ses apports en termes de dimensionnement, de validation ou d'ajustements du projet lui-même, permet aux porteurs de l'opération de négocier les bases d'une dérogation aux règles du PPRi (remblais/déblais, emprise au sol, etc.).

Les projets Rives de la Maine et Cité Fluviale Matra illustrent chacun le changement de perspective opéré depuis les années 2000. Ils illustrent particulièrement la volonté de mobiliser au travers de la démarche de projet la question du risque inondation [Barroca \& Hubert, 2008]. En effet, le projet, par sa souplesse, sa dynamique, et la multiplicité des acteurs auxquels il fait appel, favoriserait une meilleure intégration des enjeux du risque inondation par les acteurs du projet mais aussi par les populations. Si la cote de constructibilité elle-même peut être discutée par les acteurs du projet c'est surtout les dérogations et applications des règles qui vont être négociées. Ces projets, en intégrant le phénomène hydraulique, offrent une nouvelle perspective en matière d'aménagement urbain : ils agissent non plus seulement sur le bâti mais sur la morphologie du terrain, des espaces verts et publics et des constructions.

Comme les PPRi contraignent fortement les deux projets, on va assister à un jeu de «va et vient » entre les parties prenantes du projet et les services de l'État en charge de l'élaboration et de l'application de la prévention du risque inondation. L'ancien chargé de mission risques majeurs d'Angers Loire métropole explicite ce moment : «le projet de PPRI était avancé alors que le projet urbain de la Ville était encore flou. Il a fallu que la Ville précise au travers d'études limitées et rapides son projet. Un dialogue en a résulté pour que le projet de PPRI prenne en compte le projet urbain et pour que le projet urbain intègre le risque inondation, ce qu'il ne faisait pas forcément jusque-là. $\gg^{5}$. Dans les deux cas, la production d'un modèle hydraulique par un bureau d'étude a joué un rôle de médiation et permis d'apporter aux collectivités locales des arguments supplémentaires pour ses négociations avec les services d'État. La négociation se fait sur le principe que le projet doit avoir un impact hydraulique nul voire positif sur le champ d'expansion des crues et ne pas présenter d'obstacle à l'écoulement des eaux. Cela implique par exemple l'interdiction des remblais, mais cela autorise de manière dérogatoire certains mouvements de déblais/remblais sur la zone. Ainsi, la modélisation hydraulique réalisée dans le cadre du projet vise à la fois à concevoir et à démontrer la transparence hydraulique et l'impact positif ou nul des projets. Ainsi, en parallèle de la conception des deux projets étudiés, un travail important a été fait avec les services de l'État qui a permis de clarifier des points qui n'auraient pas forcément été évoqués lors de la rédaction du PPRi et donne lieu à des « doctrines d'application ».

Le second apport de la modélisation hydraulique se trouve au niveau même de la conception des projets. Dans le cas du projet Angers Rives Nouvelles sur le quartier Saint-Serge, le principe est de diviser l'espace en deux parties distinctes (Figure 2 et Figure 3 ) tout en conservant de nombreuses liaisons entres elles. Le premier espace, en bordure de la Maine, à partir du mouvement de déblais, correspond à un grand parc submersible de 20 ha appelé Parc des Confluences. Un port, deux îles (une sauvage et une destinée aux loisirs) avec deux bras de la Maine et un système de plissés étaient envisagés (Figure 3). Ce système correspond à un dispositif décliné en strates et gradins assurant en toute saison l'accessibilité au fil de l'eau et des appropriations différentes du point de vue de l'usage et du végétal (Figure 2). D'autre part, plus en retrait, l'idée est de constituer un site plus urbain : « le principe [...] est de considérer que l'activité humaine peut difficilement être en permanence sous la menace des inondations. Mais qu'en revanche elle peut côtoyer intelligemment des sites naturels d'expansion de crue ${ }^{6}$. Les déblais récupérés par la création du parc permettraient donc, par un jeu d'équilibre, d'élever le niveau du plateau urbain à $50 \mathrm{~cm}$ au-dessus des PHEC en imposant un terrassement et un nivellement général. Cela doit permettre la construction d'habitations et d'activités nouvelles sur les zones ainsi remblayées, dans une double optique de réduction de la vulnérabilité et d'accessibilité des rez-de-chaussée. "On est très attaché à ce que l'on soit de plein pied entre la rue et l'entrée de l'immeuble quitte à trouver des dispositifs parfois un peu plus complexes. »’ . La création du Parc des Confluences implique de requalifier la voie des berges sur Saint-Serge en la remplaçant par une large avenue en retrait, sur l'axe de l'ancienne voie ferrée. Cette voirie en remblais devra être transparente avec un nivellement général au-dessus de la cote des PHEC. Des commerces et

\footnotetext{
${ }^{5}$ Ex-chargé de mission risques majeurs, Angers Loire Métropole, 14/04/2014

${ }^{6}$ Ex-directeur opérationnel, SPL Angers Rives Nouvelles, 24/03/2014

${ }^{7}$ Architecte-urbaniste, Atelier Grether, 13/05/2014.
} 
des services au pied des immeubles bordant l'avenue centrale sont prévus pour assurer une mixité des fonctions et donner un caractère de centre-ville au quartier.

De l'étude portant sur le comportement hydrodynamique de la Maine, diligentée par les porteurs du projet afin d'évaluer les impacts des aménagements sur les écoulements de la rivière, il ressort que les aménagements envisagés ont des «conséquences mineures mais positives sur les conditions d'écoulement »[SCE \& CREOcéan, 2013]. Cette étude conclut que le projet s'inscrit totalement dans les «objectifs de prévention des risques d'inondation et dans l'optique d'une meilleure résilience du territoire ». Les aménagements urbains prévus permettraient ainsi, pendant l'inondation, de minimiser les désordres, d'améliorer le fonctionnement urbain et de retrouver rapidement un état normal après l'événement, éléments clés d'une résilience urbaine. Cependant, les conclusions cette étude s'appuient sur un résultat dont l'intervalle de confiance (de plusieurs centimètres) sur la ligne d'eau, compte tenu des difficultés de calage des modèles 2D, est plus important que l'effet positif de l'ordre du centimètre pour la crue de janvier 1995 sur la ligne d'eau de la Maine. La modélisation va ici plus servir à justifier le parti d'aménagement sur l'effet de l'inondation sur l'aménagement lui-même. En effet, l'étude établit que « l'emprise du champ d'expansion est réduit et limité pour le quartier Saint-Serge au périmètre du Parc des Confluences, ainsi la zone inondable pour des conditions de crues de type janvier 1995 est bien moins étendue en considérant le nouvel aménagement que dans la configuration actuelle, alors même que l'ensemble de l'urbanisation projetée ainsi que les principales voies de desserte sont rendus hors d'eau » [SCE \& CREOcéan, 2013].
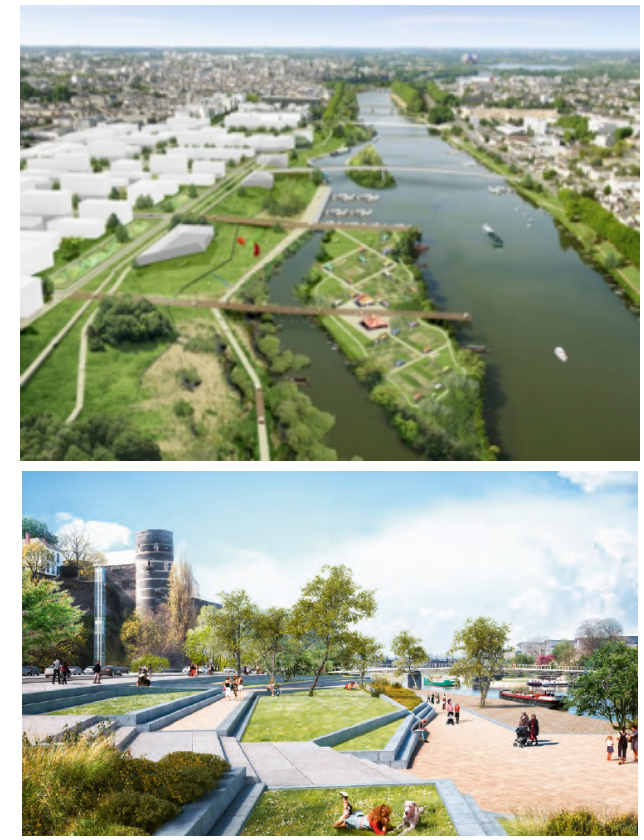

Figure 2 : Le parc et l'espace urbain $(A)$, Aménagement de type plissé (B) (crédit : Atelier Grether, 2013)

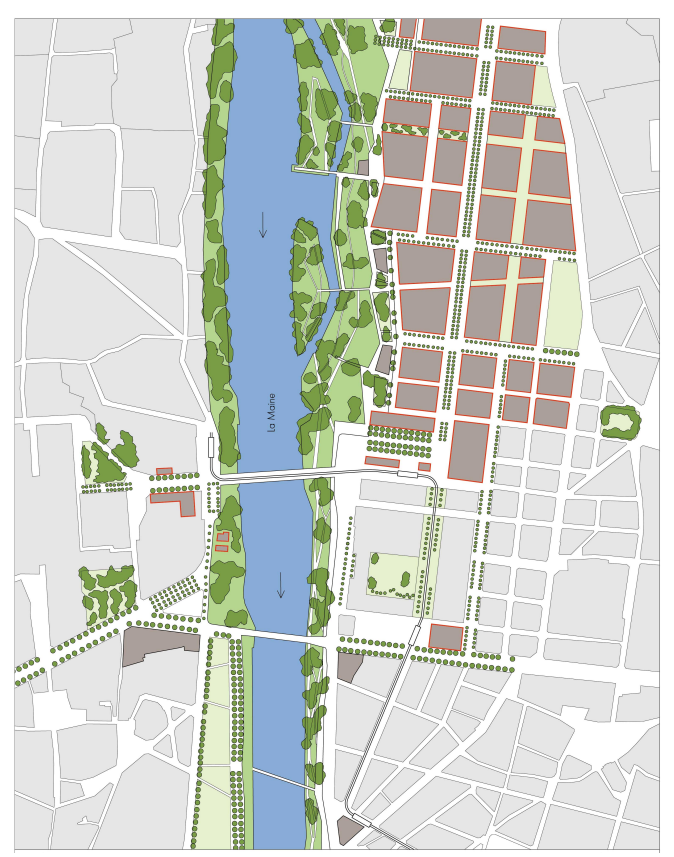

Figure 3 : Vue en plan du projet Angers Rives Nouvelles sur le quartier SaintSerge (Eric Daniel-Lacombe $\subset$ d'après

A partir de 2014, par suite d'un changement de majorité municipale, le projet est requalifié. Un projet plus modeste, notamment en conservant la voie sur berge est alors proposé par la nouvelle municipalité en association avec la même maîtrise d'œuvre. Sur le quartier Saint Serge, le projet Angers Cœur de Maine, engage un aménagement au nord de la ligne de tram dans la continuité de la ZAC St Serge 2000. Cet aménagement est toujours plus ou moins basé sur le principe d'un déblai-remblai avec la création d'un parc inondable de 5 ha.

Le Quartier Matra a été conçu lui aussi avec I'inondation comme un « affluent temporaire » (Figure 5 et Figure 6) par les équipes de I'Architecte-Urbaniste Eric Daniel-Lacombe et du Paysagiste Bernard Lassus. Si le premier projet de quartier n'avait pas intégré l'inondation, le lancement de la procédure d'élaboration du PPRi de la Sauldre a contraint à une remise à plat complète du parti pris d'aménagement initial (Figure 4). 


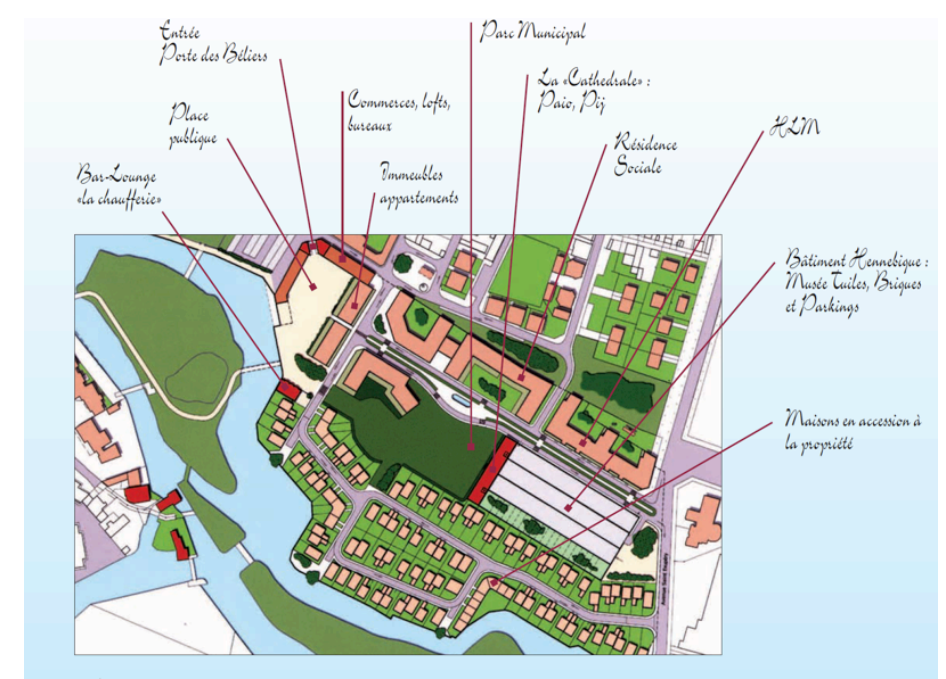

Figure 4 : Vue en plan du projet initial du promoteur sur le quartier Matra ${ }^{8}$

Les porteurs de projet, ont cherché à intégrer la crue dans la conception même du quartier, non pas en érigeant une digue, mais au contraire en faisant entrer l'eau dans le quartier, comme l'exprime bien l'architecte promoteur du quartier : « en protégeant un quartier par une digue on crée incontestablement un sentiment de sécurité, et il est fondé tant que l'eau ne passe pas au-dessus de la digue. Si au contraire l'eau monte au-delà des seuils de sécurité prévus, le déferlement des eaux produit une catastrophe : il est violent, brutal, surprend des personnes qui ne l'avaient pas vu venir, emporte les personnes qui se trouvent sur son passage et les voitures transformées en butoirs détruisant les constructions légères en aval, il arrache les arbres et ravine les terres. 》 [Daniel-Lacombe, 2016].

Il a été conçu autour d'un jeu subtil de remblais-déblais avec la mise en place du bassin de rétention paysager ainsi que de la transparence hydraulique des bâtiments, de façon à permettre l'écoulement et pour partie le stockage de l'eau dans le quartier (Figure 5). Ce parti d'aménagement s'appuyant sur des principes d'hydraulique a été dimensionné par la modélisation hydraulique.

\footnotetext{
${ }^{8}$ Extrait du DOSSIER "Romo 1 : un nouveau quartier se dessine" in Bulletin municipal de Romorantin de janvier 2008.
} 

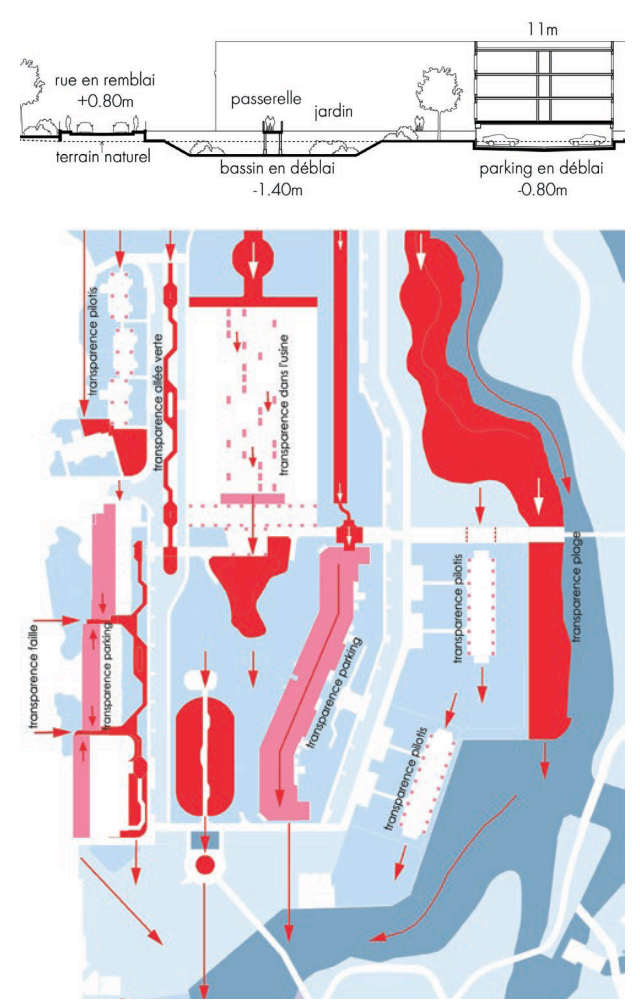

Figure 5 : Coupe des mouvements de déblaisremblais entre le bassin de rétention, les parkings et la voirie $(A)$; Vue en plan des transparences hydrauliques $(\rightarrow$ ) mise en place sur la zone ainsi que les zones de rétention (

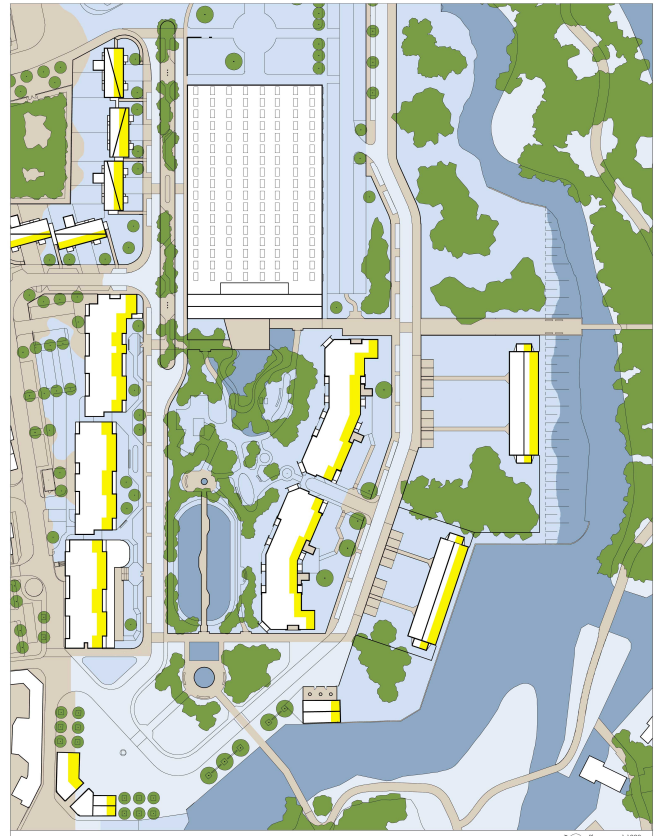

Figure 6 : Vue en plan de la cité fluviale Matra à Romorantin (Eric

Ces deux projets s'inscrivent bien dans la doctrine de l'aménagement urbain : « utiliser les zones inondables dans l'aménagement urbain, c'est à la fois faire œuvre d'urbanisme, gérer le risque et aussi protéger l'environnement. » [Dégardin \& Gaide, 1999]. Les notions d'expansion des crues, de transparence hydraulique ou de rétention des eaux se trouvent au cœur de la conception de ces projets et non réduites à des contraintes imposées par les règles d'urbanisme et de prévention du risque. On passe ainsi d'une définition par la règle de la morphologie urbaine en zone inondable qui contraint la construction des bâtiments indépendamment les uns des autres à une approche plus globale par le projet qui cherche à englober l'ensemble d'un quartier. Le processus devient plus important que les réglementations ou procédures [Ingallina 2002 ; Novarina 2003]. Dans ces deux cas, la modélisation a permis aux porteurs de projet de négocier avec les services de I'Etat l'application du PPRi. Les morphologies urbaines de ces projets intègrent la crue et sont le résultat d'une conception en lien avec des travaux de modélisation hydraulique. Si le projet Angers Rives Nouvelles n'a pu être réalisé, la cité fluviale de Matra à Romorantin-Lanthenay a été construite et a même subi une submersion supérieure aux PHEC sans subir de dégâts majeurs quand la ville historique subissait des dégâts très importants ${ }^{9}$. Cet évènement exceptionnel montre bien « I'aptitude à faire face » de ce quartier, qui passe par un «fonctionnement en mode dégradé » pour retrouver rapidement un état de «fonctionnement acceptable » [Serre, 2011 ; Barroca \& al., 2013]. Si ces deux projets présentent incontestablement le mérite de décentrer l'approche en matière de risque de la planification vers la conception grâce à la modélisation hydraulique, ne constituent-ils pas de simple adaptation technique à un problème complexe qui dépasse cette dimension ?

\section{AU-DELA DE LA TECHNIQUE, LA MODELISATION HYDRAULIQUE, CONDITION D'UNE RESILIENCE URBAINE?}

En quoi, la modélisation hydraulique peut-elle participer à la recherche de la résilience urbaine ? D'abord en tant qu'outil technique, elle permet de projeter les effets d'un aménagement sur un phénomène, contribuant ainsi à la recherche d'une solution technique résiliente. Cet outil contribue à définir les conditions de l'adaptation aux phénomènes exceptionnels, objectif d'une résilience portée par le management des risques, et qui sous-tend une approche dynamique et donc une rupture avec l'approche

9 Cf. article Sibylle Vincendon «Inondations: un quartier touché mais pas coulé » publié sur www.Liberation.fr le 27 juin 2016. 
actuelle plutôt «statique » des documents de planification. Cela permet, quand elle est mobilisée pour la conception des projets d'aménagements urbains, de dé-compartimenter les différents acteurs de la ville qu'ils soient publics ou privés. C'est aussi un outil de connaissance qui peut être mobilisé dans le cadre de la participation des acteurs [Barroca, 2006] essentielle à la mise en place d'une résilience urbaine face au risque inondation. Ainsi, la modélisation permet d'apporter une réponse aux freins et difficultés de la mise en œuvre d'une stratégie d'adaptation du système urbain liés notamment à la compartimentation des acteurs [Barroca et al., 2013 ; Barroca et al., 2012 ; Serre, 2011]. Elle s'inscrit alors comme un média de négociation, et non plus comme la ressource d'action d'un acteur.

Le travail de co-conception du quartier Matra mobilisant la modélisation hydraulique a permis de produire un quartier résilient à l'inondation. Cette résilience a été effective lors de la crue de mai-juin 2016. Eric Daniel-Lacombe, concepteur du projet dans le dossier de presse post-crue illustre bien cet aspect en expliquant que « Le quartier a été conçu pour permettre aux habitants de voir l'eau monter, de disposer du temps nécessaire à l'évacuation de leurs voitures, de prendre la mesure de la montée des eaux et de décider de rester ou quitter leurs logements. Le quartier a été conçu pour stocker une partie de l'eau dans un bassin de rétention, ralentir le flot supplémentaire et le canaliser afin qu'il retourne à la rivière en aval, moins turbulent qu'à son entrée. Les niveaux des jardins, des voiries, des garages et des planchers d'appartement sont différents et permettent des départs en sécurité à l'initiative des habitants tant qu'une cote supérieure de $30 \mathrm{~cm}$ à la cote de la crue centennale n'est pas atteinte. Au-delà les logements disposent de terrasses accessibles par les bateaux des pompiers, ceci parce que les eaux ne sont pas dramatiquement turbulentes. Le ralentissement des flux assure la sécurité des habitants en toutes circonstances. 》 [DanielLacombe, 2016]. Face à un évènement très exceptionnel, puisque la cote atteinte durant la crue de la Sauldre du printemps 2016 était supérieure de plusieurs dizaines de centimètre aux PHEC, le quartier, inondé (Figure 7), s'est comporté comme prévu, laissant le temps aux habitants d'évacuer et de mettre les véhicules à l'abri. Aucun plancher habitable n'a été inondé. Les niveaux d'eau, bien qu'ayant dépassés les PHEC n'ont pas atteint la cote plancher. Le principe d'une surcote de $50 \mathrm{~cm}$ par rapport aux PHEC imposée par les services de l'État dans le PPRi a été ici salutaire. L'eau s'est retirée en quelques jours, permettant le nettoyage des rues, parking, etc. La présence de gendarmes dans les immeubles a facilité la coordination entre les habitants et la mise en sécurité des équipements. Les habitants du quartier ont suivi la montée des eaux, tant annoncée.

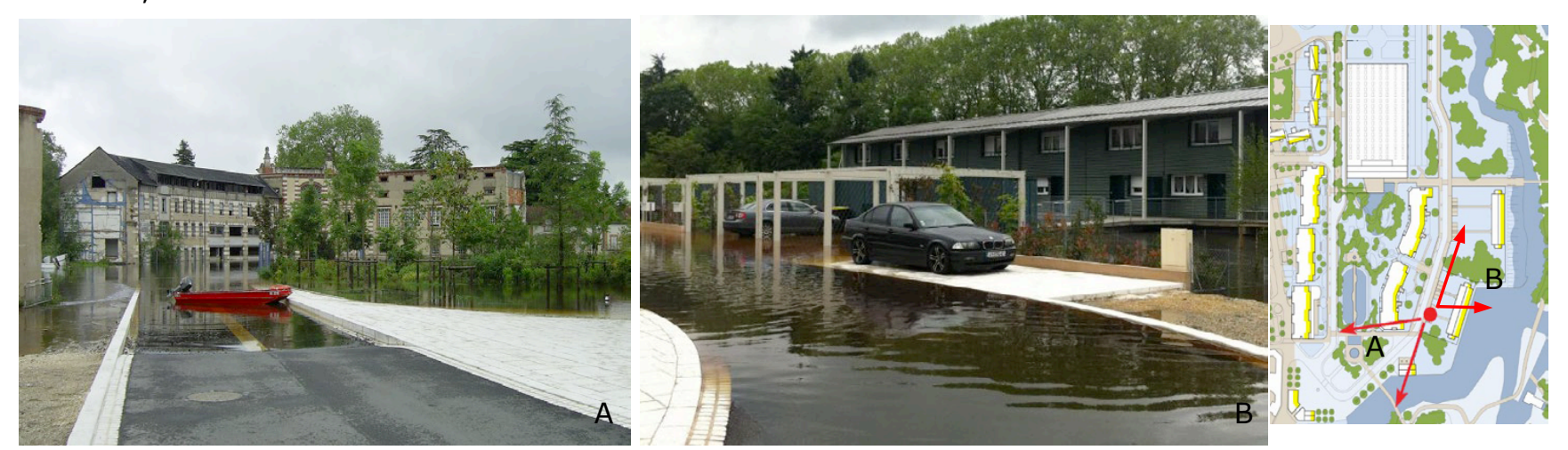

Figure 7 : Photographies représentant I'inondation lors de la montée des eaux dans le quartier (Crédit Eric Daniel-Lacombe C)

Si longtemps la modélisation hydraulique était peu discutée et servait de légitimation à l'action publique, elle est de plus un plus un support de discussion et de négociation. La modélisation hydraulique s'inscrit dans une certaine mesure dans le processus de « mise en ordre » de l'action collective locale permettant aux acteurs de se coordonner. Les processus de négociation sont des moments organisateurs de cette action collective. Elle met en scène un ensemble interdépendant d'acteurs individuels et/ou collectifs, qui bien qu'étant en concurrence sur la définition des «problèmes » et sur l'élaboration des « solutions » [Friedberg, 1993 : 177], vont trouver avec la modélisation hydraulique un support de discussion. Les résultats de cette modélisation sont à la fois discutés et constituent une base de discussion sur laquelle les acteurs vont élaborer une décision plus ou moins collective. Ce processus est complexe. Selon les acteurs impliqués, la modélisation peut être confrontée à d'autres formes de savoirs ou au contraire être un élément de convergence en matière de savoirs. Dans la première situation, la vraisemblance des résultats peut être mis en doute au regard de la connaissance des certains acteurs (mémoire historique, connaissance du terrain, etc.), alors que dans la seconde, la robustesse de la modélisation sera plus discutée que ses résultats. La modélisation, si elle ne constitue pas comme on pourrait l'attendre à première vue un élément objectif intégré par les différents acteurs, elle est toutefois une base de négociation, plus ou moins instrumentalisée, pour ces acteurs.

In fine, la modélisation participe à construction d'une résilience urbaine au travers de la prise en charge du risque inondation. La gestion du risque inondation apparaît comme n'étant plus une simple politique de prévention portée par l'État mais devenant une des données au cœur du processus de décision collective [Barroca \& al., 2013]. La question de la gestion du risque est ainsi étendue aux problématiques 
d'aménagement urbain, non pas par l'intégration des règles mais par l'intégration du phénomène, cette dernière étant permise par la modélisation. La modélisation hydraulique participe ainsi à la fois à la production de règles secondaires de mise en œuvre de l'action publique [Lascoumes \& Le Galès, 2012] et à la prise en charge du risque inondation. Le risque inondation apparaît comme un liant territorial et est alors examiné comme une composante à part entière du système socio-territorial [November, 2008 ; Reghezza 2006].

\section{CONCLUSIONS}

Au travers de ces deux cas, nous montrons que la modélisation hydraulique est mobilisée par différents acteurs, d'abord les services de l'Etat dans I'optique de réguler au travers de l'élaboration des PPRi, ensuite par les acteurs du projet pour négocier l'application de ces règles et de concevoir le projet. La modélisation hydraulique participe du renouvellement des activités de conception. Les architectes-urbanistes qui prennent en charge les questions de conception en lien avec la contrainte inondation voient leur champ de compétences, leurs savoirs et leurs savoir-faire réinterrogés [Gralepois \& Guevara, 2015]. Le périmètre des acteurs se redessine et cela aux différentes échelles, de la ville au bâtiment en passant par le quartier. D'une part, si les professions traditionnellement impliquées, architecte, urbaniste, paysagiste, dans ces activités doivent démontrer leur capacité à intégrer la question du risque inondation, ce qui peut passer par le développement de nouvelles compétences de nouvelles approches autrefois à la marge de leur pratique. On constate un phénomène d'apprentissage important de ces professionnels, comme nous avons pu l'observer dans nos deux cas. D'autre part, les hydrauliciens apparaissent comme de nouveaux acteurs des projets du fait qu'ils détiennent une expertise spécifique et jouent, à ce titre, un rôle de plus en plus important dans les activités de conception des projets d'aménagement en zone inondable. Ils n'interviennent plus seulement comme des sous-traitants ponctuels sur commande, mais deviennent des membres significatifs des groupements de maîtrise d'œuvre et sont associés à la définition des principes et partis-pris d'aménagement. Au-delà de la conception, les deux cas étudiés montrent bien en quoi le projet d'aménagement urbain en zone inondable est subordonné à la négociation de l'application de la règle de planification en vigueur (PPRi). Cette négociation se fonde pour une grande partie sur un argumentaire technique, celui de l'hydraulique. Pour cela, l'expertise des bureaux d'étude est déterminante. Cette expertise permet aux porteurs de projet de démontrer que l'impact de leur projet d'aménagement est nul voire positif sur les écoulements et ainsi de pouvoir déroger aux règles d'emprise et de cote plancher qui s'imposent aux constructions dans cette zone. La participation de ces spécialistes à la négociation et, du même coup, aux choix et partis-pris d'aménagement, ouvre des possibilités jusque-là inenvisageables. En retour, les acteurs de la planification se trouvent eux-aussi impliqués plus largement dans les activités de conception qu'ils saisissaient auparavant sous l'unique angle de la régulation. De nouveaux principes d'aménagement peuvent ainsi émerger des échanges entre ces acteurs et constituer potentiellement une source d'innovation tant technique que sociale. Le quartier inondable de la Cité Fluviale Matra à Romorantin-Lanthenay en témoigne. La modélisation hydraulique, n'est pas uniquement un outil technique, elle constitue aussi une base pour les échanges entre les différents acteurs (élus, services de l'État, architecte-urbaniste, paysagiste, etc.). Cette dynamique est permise par l'intégration dans la maîtrise d'œuvre de bureaux d'études hydraulique et par un changement d'approche de l'architecte-urbaniste et du paysagiste [Rode \& al., 2018]. Ainsi, des formes de relations et de coordination entre les différents acteurs émergent de la mobilisation de la modélisation hydraulique et entraînent une recomposition de I'action collective locale.

Un effet pervers pourrait être de mettre en place une croyance en I'infaillibilité de l'outil technique de la modélisation, à l'instar des dispositifs de protection (digue, etc.) [Degoutte, 2005], la « construction collective d'une confiance en la protection qui empêche la constitution d'une culture locale du risque » [Durand 2014]. Cependant, le fait de concevoir avec la modélisation hydraulique les quartiers en zone inondable peut permettre a contrario de construire cette culture du risque, car on ne cherche pas à protéger le quartier de l'eau mais au contraire à l'intégrer aux conditions d'écoulement en crue. Cela peut permettre de développer d'une certaine manière une culture locale du risque à long terme. Cela nécessite notamment de s'appuyer sur les résultats de la modélisation et les dispositifs techniques d'intégration de l'inondation (bassin de rétention et zone d'expansion de crue paysager, dispositifs de transparence hydraulique, etc.) pour l'information des habitants. La modélisation hydraulique, participe dans une certaine mesure à l'émergence de la société du risque et d'une modernité réflexive [Beck, 2001 : 335].

Cette approche en matière de conception des projets d'aménagement par la modélisation pourrait permettre, dans une perspective d'adaptation au changement climatique de mieux prendre en compte l'augmentation des événements hydrologiques extrêmes. C'est dans cet esprit que s'inscrit la Directive européenne Inondation de 2007 en imposant la définition d'un scénario « extrême » pour les Territoires à Risque Inondation, alors que les Plus Hautes Eaux Connues (PHEC) déterminée dans les PPRi correspondent à une "crue moyenne" au sens de cette Directive.

Enfin, le développement d'une culture de l'incertitude en matière de modélisation des inondations mais aussi de décision est déterminant. Face à ces phénomènes hydrologiques extrêmes de plus en plus fréquents, aggravés par une urbanisation importante des zones inondables qui limite l'expansion des crues, 
la modélisation pourrait permettre aux décideurs de mieux appréhender le risque d'inondation et la prise de décision dans des situations incertaines, tant au niveau de la planification que de l'aménagement.

\section{REMERCIEMENTS}

Tous mes remerciements vont aux membres du projet PRECIEU, principalement Mathilde Gralepois, Sylvain Rode, Eric Daniel-Lacombe, Alexis Moreau et Sofia Guevara.

\section{REFERENCES}

Atelier Grether (2013). Plan-guide Angers Rives Nouvelles, publié en mai 2013

Barroca B. (2006). Risque et vulnérabilités territoriales : Les inondations en milieu urbain. Thèse de doctorat de l'Université de Marne la Vallée, Champs-sur-Marne, 296 p. + annexes.

Barroca B. \& Hubert G. (2008). Urbaniser les zones inondables, est-ce concevable ? Développement durable et territoires [En ligne], Dossier 11 | 2008.

Barroca B., DiNardo M. et Mboumoua I. (2013). De la vulnérabilité à la résilience : mutation ou bouleversement ? EchoGéo [En ligne], 24 | 2013.

Barroca B., Serre D. \& Diab Y. (2012). —Le concept de résilience à l'épreuve du génie urbain. Vertigo - la revue électronique en sciences de l'environnement [En Ligne], 12.

Beck U. (2001). La société du risque - Sur la voie d'une autre modernité. Paris, Éditions Aubier.

Bernardara P. et Barroca B. (2005). - Integrated tools for flood risk estimation. In Université de l'eau, Conseil Général du Val de Marne, World Wide Workshop for Young Environmental Scientist, Paris, 10-13 May 2005, p. 119-126.

Daniel-Lacombe E. (2016). Quand l'architecture permet de prévenir les crues et d'y résister sans dommage en période d'inondation, communiqué de presse, juin 2016.

Dégardin F. \& Gaide P.-A., (1999). Valoriser les zones inondables dans l'aménagement urbain. Éditions du Certu, Collection Dossiers, $231 \mathrm{p}$

Degoutte G. (2005). Peut-on endiguer les risques dus aux digues ? Avant-propos, Ingénieries Eau-Agriculture-Territoires, Editions Quae, $n^{\circ}$ spécial 2005, 212p

Durand, S. (2014). Vivre avec la possibilité d'une inondation ? Ethnographie de I'habiter en milieu exposé... et prisé, thèse de doctorat en sociologie, Aix-Marseille Université.

Friedberg E. (1997). Le pouvoir et la règle, dynamiques de l'action organisée, Seuil.

Gralepois M. et Guevara S. (2015). L'adaptation aux risques d'inondation façonnée par les métiers de la ville, Développement durable et territoires [En ligne], Vol. 6, n³ I Décembre 2015.

Ingallina P. (2002). Le projet urbain, Annales de Géographie, vol. 111, n 623, pp. 98, Lascoumes P., Le Galès P. (2012). Sociologie de l'action publique. (2e édition), Armand Colin, coll. « $128 », 2012,128$ p.

Novarina G. (2003). Plan et projet. L'urbanisme en France et en Italie, Anthropos, coll. «illes », pp. 233.

November V. (2008). Spatiality of risk, Environment and Planning A, no 40, p. 15231527. 
Reghezza M., Rufat S., Djament-Tran G., Le Blanc A.\& Lhomme S. (2012). What Resilience Is Not: Uses and Abuses. Cybergeo: European Journal of Geography [En ligne], document 621, mis en ligne le 18 octobre 2012

Reghezza M. (2006), Réflexions sur la vulnérabilité métropolitaine. La métropole parisienne face au risque de crue centennale. Thèse de doctorat, Université de Paris XNanterre, $384 \mathrm{p}$.

Rode S. (2017). Reconquérir les cours d'eau pour aménager la ville, Cybergeo : European Journal of Geography [En ligne], document 806, mis en ligne le 30 janvier 2017.

Rode S., Guevara S. \& Bonnefond M. (2018). Resilience in urban development projects in flood-prone areas: a challenge to urban design professionals, Town Planning Review TPR, 89 (2) 2018

SCE \& CREOcéan (2013). Etude hydrodynamique et hydrosédimentaire du projet Angers rives nouvelles.

Serre, D. (2011). La ville résiliente aux inondations - Méthodes et outils d'évaluation. Mémoire d'Habilitation à Diriger les Recherches, Université Paris-Est, 173 p. 